\title{
Acoustic bubble sizing by combination of subharmonic emissions with imaging frequency
}

\author{
T.G. Leighton, R.J. Lingard, A.J. Walton and J.E. Field \\ Cavendish Laboratory, Madingley Road, Cambridge CB3 OHE, UK
}

Received 8 October 1990; revised 12 December 1990

\begin{abstract}
The non-linear response of a bubble to an acoustic field results in the scattering of sum- and difference-frequencies when two frequencies are incident. This has in the past been used to size bubbles, since it is only when the pump frequency, $\omega_{p}$, is near the bubble resonance that the non-linear effects are observed. In addition, the subharmonic frequency emitted by the bubble can combine with the imaging frequency, $\omega_{i}$, to produce scattered signals at $\omega_{\mathrm{i}} \pm\left(\omega_{\mathrm{p}} / 2\right)$. In this study, the ability of these signals to accurately size and resolve bubbles, both singly and in arrays, has been studied.
\end{abstract}

\section{Keywords: bubble sizing; non-linear acoustic scattering; pump frequency; imaging frequency}

A gas bubble in a liquid may be forced into volume pulsations by an acoustic field. The equations describing the bubble motion are highly non-linear, one of the commonest being the Rayleigh-Plesset equation ${ }^{1}$

$$
\begin{aligned}
& R \ddot{R}+3 \dot{R}^{2} / 2=(1 / \rho)\left\{\left(P_{0}+2 \sigma / R_{0}-P_{\mathrm{v}}\right)\left(R_{0} / R\right)^{3 \kappa}-2 \sigma / R\right. \\
& \left.\quad-4 \mu \dot{R} / R-P_{0}-P(t)\right\}
\end{aligned}
$$

where $R$ is the bubble radius and $R_{0}$ its equilibrium value; $\sigma, \rho$ and $\mu$ are, respectively the surface tension, density and viscosity of the liquid; $P_{0}$ is the hydrostatic pressure; $P(t)$ is the time-varying pressure component (i.e. the driving term); $P_{\mathrm{v}}$ is the vapour pressure within the bubble; and $\kappa$ is the polytropic index of the gas. The value of $\kappa$ lies between 1 and $\gamma$, the ratio of the specific heats at constant pressure and volume, depending on whether the gas behaves isothermally, adiabatically or in some intermediate manner. When the surface tension and viscosity terms in the Rayleigh-Plesset equation are deemed negligible and $P(t)$ is set to zero, the formulation approximates to the resonant oscillations of an unforced bubble, at a frequency

$v_{\mathrm{r}}=\left(1 / 2 \pi R_{0}\right)\left(3 \kappa P_{0} / \rho\right)^{1 / 2}$

This equation was first derived by Minnaert $^{2}$ who assumed simple harmonic oscillation of the bubble wall. For air bubbles in water under atmospheric pressure, Equation (2) reduces to

$v_{\mathrm{r}} R_{0} \approx 3 \mathrm{~Hz} \mathrm{~m}$

Equation (2) allows bubbles to be sized by measurement of the frequencies they emit when mechanically excited ${ }^{3,4}$. Bubble sizing has also been attempted by the use of incident acoustic signals, utilizing resonance excitation ${ }^{5}$, Doppler techniques ${ }^{6}$ and the stimulation of the second harmonic ${ }^{7}$, but these techniques all have limitations ${ }^{8}$. For example, the signal scattered from a small, resonance bubble in resonance excitation can be weaker than the signal from a larger, non-resonant bubble. It may be difficult to distinguish a cluster of small bubbles from a single large one using Doppler. Spatial resolution is poor with second harmonic generation.

Bubble sizing has a wealth of applications, including investigations into decompression sickness ${ }^{9,10}$, contrast echocardiography ${ }^{11}$ and the monitoring of pressure fluctuations $^{8}$ and of the sodium coolant in nuclear reactors ${ }^{12}$. Attempts have been made to detect bubbles acoustically in high pressure vessels ${ }^{13}$ and in canine cardiovascular systems ${ }^{14}$.

Newhouse and Shankar ${ }^{15}$ introduced the technique of bubble sizing using the scattered signals when a bubble is insonated with a pump frequency $\omega_{\mathrm{p}}$ and an imaging frequency $\omega_{\mathrm{i}}$. When $\omega_{\mathrm{p}}$ is near the bubble resonance, the amplitude of oscillation of the bubble wall is large and the bubble behaves non-linearly. The general response, $Y$, of a bubble (for example, its wall motion or the scattered signal from it) may therefore be expressed as a power series

$$
Y(t)=\xi_{1} P(t)+\xi_{2} P^{2}(t)+\xi_{3} P^{3}(t)+\xi_{4} P^{4}(t)+\ldots
$$

where $\xi_{1}, \xi_{2}, \xi_{3}$ and $\xi_{4}$ are constants. If the driving pressure is $P(t)=P_{\mathrm{p}} \cos \omega_{\mathrm{p}} t+P_{\mathrm{i}} \cos \omega_{\mathrm{i}} t$, then the quadratic term in Equation (4) gives rise ${ }^{16}$ to signals at $\omega_{\mathrm{i}} \pm \omega_{\mathrm{p}}$. The further $\omega_{\mathrm{p}}$ is from the bubble resonance, the more the bubble is like a linear oscillator and the smaller the quadratic contribution. Shankar et al. ${ }^{8}$ therefore took the appearance of the $\omega_{\mathrm{i}}+\omega_{\mathrm{p}}$ frequencies to indicate that $\omega_{\mathrm{p}}$ equalled the bubble resonance, and so had a measure of the bubble size.

A stable bubble driven in an intense acoustic field also emits a subharmonic at half the driving frequency when the bubble is driven near resonance ${ }^{17,18}$. In the present study, the combination of this subharmonic (at $\left.\omega_{\mathrm{p}} / 2\right)$ 
with an incident imaging frequency, $\omega_{i}$, generated signals at $\omega_{\mathrm{i}} \pm\left(\omega_{\mathrm{p}} / 2\right)$. The sharpness of the $\omega_{\mathrm{i}} \pm\left(\omega_{\mathrm{p}} / 2\right)$ response was compared with that of the $\omega_{i} \pm \omega_{p}$ frequencies, and the relative abilities of the two sets of signals to elucidate bubble resonance and size were assessed. Acoustic measurements of bubble size obtained in this manner were then used to estimate the value of the polytropic index of air, helium and propane in these processes. Finally, the techniques were used to resolve acoustically bubble arrays.

\section{Experimental details}

The experiment is performed in a polymethylmethacrylate (PMMA) tank $\left(25 \times 25 \times 50 \mathrm{~cm}^{3}\right)$, filled with water and lined with acoustic absorber (Figure 1). A $3.35 \mathrm{MHz}$ continuous-wave imaging signal was generated at 0.05 $\mathrm{W} \mathrm{cm}^{-2}$ by a Therasonic 1030 ultrasound generator (Electro-Medical Supplies) through an acoustic window. The pump signal was produced in the water tank by a submerged $85 \mathrm{~mm}$ Mylar cone speaker (Radio Spares type 248-325), which was driven by a signal generator (Brookdeal type 471) via a paralleled amplifier (Quad type 405-2). The voltage applied to the speaker was kept constant for each separate bubble; however it was varied between bubbles in the range $1.00-5.23 \mathrm{~V}_{\text {rms }}$ to obtain a large scattered signal.

Shankar et al. ${ }^{8}$ measured the frequency of the scattered sum- and difference-signals. However since the sum- and difference-frequencies are generally about 1000 times the pump frequency, to obtain a $1 \%$ accuracy in the pump (and therefore in the measurement of the bubble resonance) would require a stability of $0.001 \%$ in the imaging frequency if the sum and difference components were measured in the manner described by Shankar et al. In the present experiment, the audio pump frequency was measured directly using a frequency counter, to prevent drift in the frequency of the signal sources from introducing a systematic error.

To prevent direct coupling between the speaker and the transducer, their axes were angled at $135^{\circ}$ in the same horizontal plane. The bubble, blown underwater by a drawn glass pipette, was held on a wire of diameter $0.5 \mathrm{~mm}$ at the intersection of the transducer axes. To aid attachment of the bubble, the wire was coated with a thin film of paraffin wax. With no bubble present, the control experiment showed that sound was not scattered non-linearly by the wire alone.

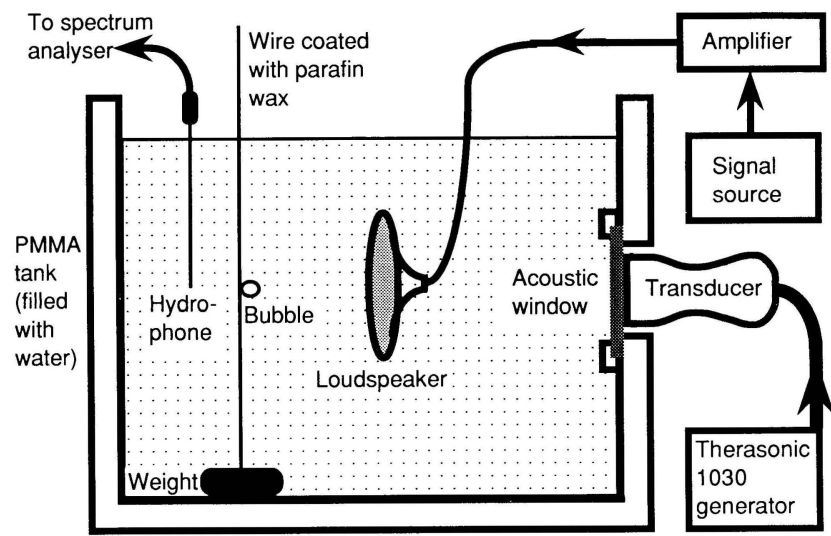

Figure 1 Apparatus used to insonate a bubble with two frequencies
A needle hydrophone (Dapco NP10-3) detected the scattered megahertz acoustic signal, which was then processed by a spectrum analyser (Marconi Instruments type TF 2370). The active element of the needle hydrophone was $10 \mathrm{~mm}$ radially away from the bubble and at the same height. The hydrophone had been calibrated for this directional mode. An example of the analyser output can be seen in Figure 2: the $\omega_{\mathrm{i}} \pm\left(\omega_{\mathrm{p}} / 2\right)$ pressures were averaged, as were the values of $\omega_{\mathrm{i}} \pm \omega_{\mathrm{p}}$. For each setting of the pump frequency, the spectrum analyser swept across the whole of the monitored frequency range. The pump frequency was then increased by between 20 and $50 \mathrm{~Hz}$, as required and the whole spectrum measured afresh.

An estimate of the bubble size could be gained by microscopy for comparison with acoustic measurements of the bubble radius. A weighted average of the long and short axes of the bubble was taken for those bubbles distorted from the spherical. The response curves for the $\omega_{i} \pm \omega_{p}$ and the $\omega_{i} \pm\left(\omega_{p} / 2\right)$ signals were compared for single bubbles, whose sizes were then measured by microscopy: the plot of resonance frequency against size could then be compared with the theoretical predictions of Equation (2) and conclusions drawn regarding the polytropic index of air, helium and propane. Helium has a wide range of potential values for $\kappa$ ( 1 to $\gamma=1.63$ ) so that the extent to which the process is isothermal or adiabatic should be clear from the results. Propane has a narrow range of $\kappa(1$ to $\gamma=1.13)$; by reducing the uncertainty caused by $\kappa$, the other sources of error in the result can be examined.

An array of four bubbles (Figure 3 illustrates the geometry of the experiment) was acoustically resolved using the scattered $\omega_{\mathrm{i}} \pm \omega_{\mathrm{p}}$ and $\omega_{\mathrm{i}} \pm\left(\omega_{\mathrm{p}} / 2\right)$ signals. Finally, high speed photography was used to image the bubble on the wire at 6000 frames per second (f.p.s.) using a Hadland Hyspeed camera.

\section{Results}

Figure 4 shows a typical response curve for the $\omega_{\mathrm{i}} \pm \omega_{\mathrm{p}}$ and $\omega_{\mathrm{i}} \pm\left(\omega_{\mathrm{p}} / 2\right)$ signals emitted from single air bubbles. The bubble used for the graph in Figure $4 b$ had a

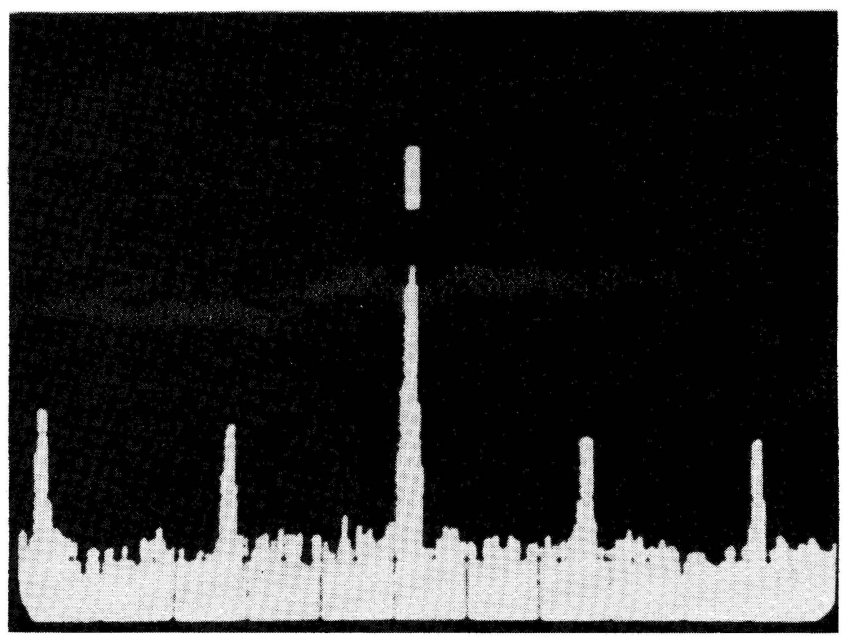

Figure 2 Typical output of the spectrum analyser, with frequency measured along the bottom. From left to right, the peaks correspond to the frequencies $\omega_{i}-\omega_{p}, \omega_{i}-\left(\omega_{p} / 2\right), \omega_{i}, \omega_{i}+\left(\omega_{p} / 2\right), \omega_{i}+\omega_{p}$. The value of $\omega_{\mathrm{p}}$ is $4.950 \mathrm{kHz}$ 


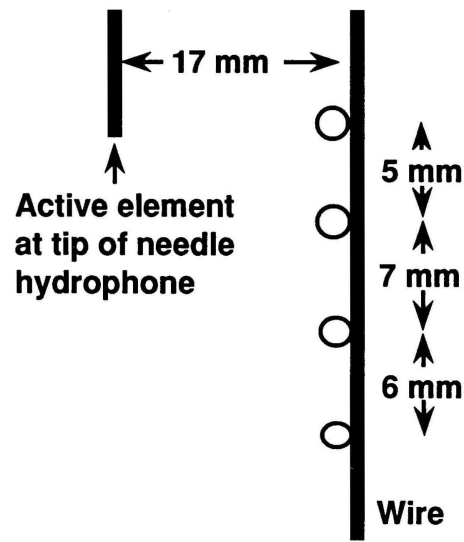

Figure 3 Geometry of the four-bubble array
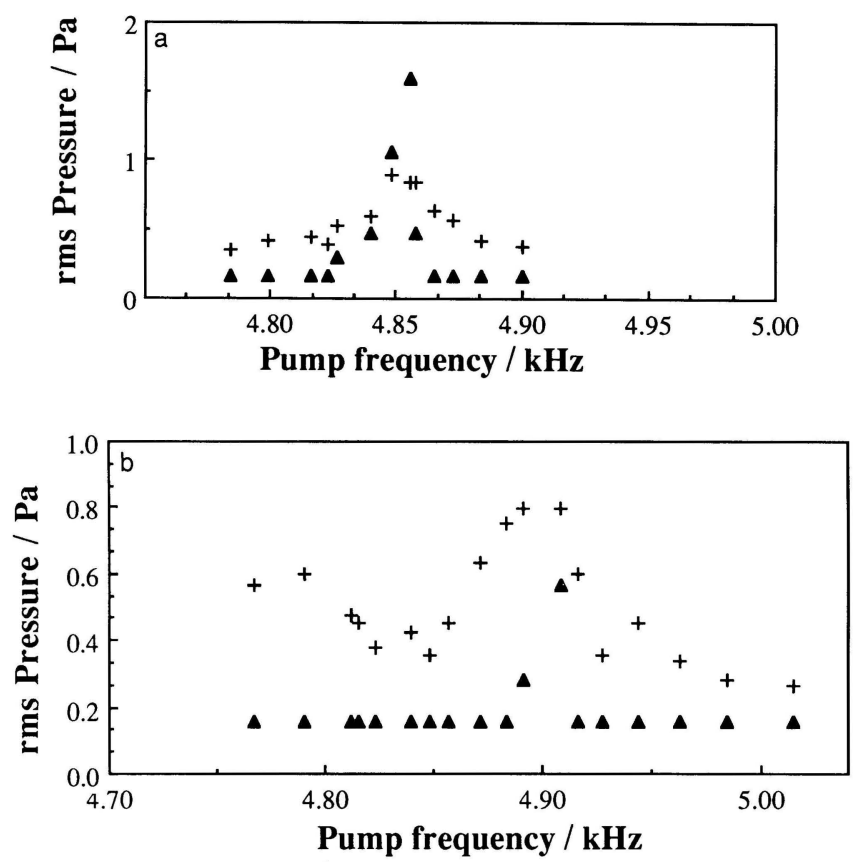

Figure 4 Response curves of single bubbles, comparing the $\omega_{\mathrm{i}} \pm \omega_{\mathrm{p}}(+)$ and the $\omega_{\mathrm{i}} \pm\left(\omega_{\mathrm{p}} / 2\right)(\boldsymbol{\Delta})$ emissions. (a) Response of air bubble in water. (b) Response of air bubble with optically measured radius of $0.645+0.005 \mathrm{~mm}$; here the peak is at $4.90 \pm 0.02 \mathrm{kHz}$ when the atmospheric pressure was $0.1000 \mathrm{MPa}$ and the water density $1021.5 \mathrm{~kg} \mathrm{~m}^{-3}$

measured radius of $0.645 \pm 0.005 \mathrm{~mm}$. In both graphs the peak corresponding to the $\omega_{i} \pm\left(\omega_{\mathrm{p}} / 2\right)$ response is clearly sharper. Beyond the range of the plots, the scattered signals were at the level of the noise. Data from many such curves are used in Figure 5 to test Equation (2), by plotting the bubble resonance frequency against the optically measured radius. These data points should lie between the predicted limits of $\kappa=1$ and $\kappa=\gamma$, marked as lines on the graph for each gas: the solid lines correspond to $\kappa=1$ (the isothermal case), $\kappa=1.13$ (propane), $\kappa=1.4$ (air) and $\kappa=1.63$ (helium).

Figure 6 compares the response curves at $\omega_{\mathrm{i}} \pm \omega_{\mathrm{p}}$ and $\omega_{\mathrm{i}} \pm\left(\omega_{\mathrm{p}} / 2\right)$ for the linear array of four bubbles illustrated in Figure 3. Finally, Figure 7 shows the bubble on the wire, subjected to the acoustic field: the bubble wall is modulated by oscillating ripples. It is these ripples which cause the surface of the bubble to 'shimmer', as seen by the naked eye, when the pump frequency approaches the bubble resonance.

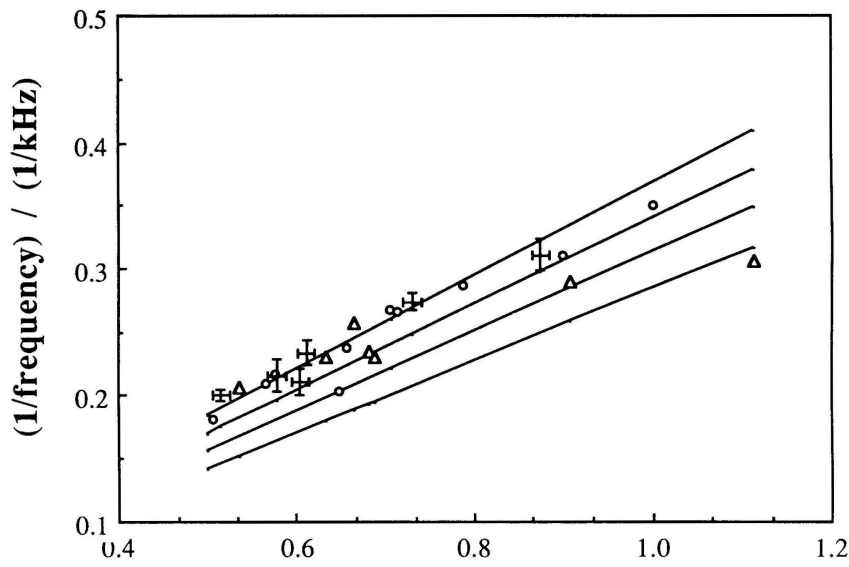

\section{Bubble radius / $\mathrm{mm}$}

Figure 5 Peak frequency of response, shown as a function of optically measured bubble radius. Data are shown for bubbles of: + , propane; $O$, air; and $\triangle$, helium in water. - , Value of the polytropic index, showing the isothermal limit and the limit for $\gamma$ of each gas. From the top, $\kappa$ has values $1.00,1.13,1.40$ and 1.63

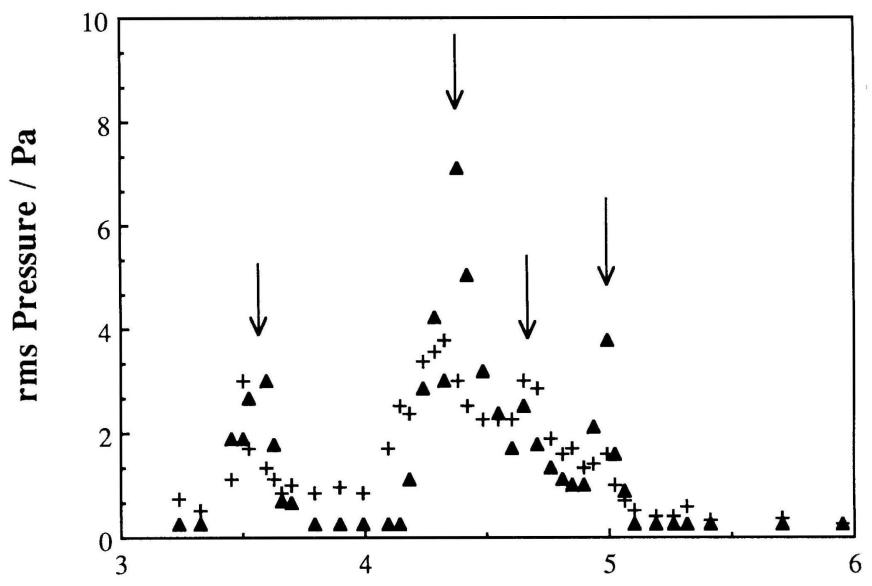

\section{Pump frequency / $\mathrm{kHz}$}

Figure 6 Response curve for four-bubble array, comparing the $\omega_{\mathrm{i}} \pm \omega_{\mathrm{p}}(+)$ and $\omega_{\mathrm{i}} \pm\left(\omega_{\mathrm{p}} / 2\right)(\boldsymbol{\Delta})$ emissions. The resonance of each bubble is arrowed (at 4.990, 4.654, 4.384 and $3.597 \mathrm{kHz}$ ). The $4.990 \mathrm{kHz}$ bubble had an optically measured radius of $0.480 \pm 0.005 \mathrm{~mm}$. The atmospheric pressure was $0.1026 \mathrm{MPa}$ and the water density $1021.4 \mathrm{~kg} \mathrm{~m}^{-3}$

\section{Discussion}

The scattered $\omega_{\mathrm{i}} \pm\left(\omega_{\mathrm{p}} / 2\right)$ signal tends to be sharper than the $\omega_{\mathrm{i}} \pm \omega_{\mathrm{p}}$ signal, making it a better tool for the resolution and sizing of bubbles. The maximum is usually greater and the half-height width (averaged over the 23 resonance curves used to develop Figure 5) is $40 \pm 20 \%$ that of the $\omega_{i} \pm \omega_{p}$ curves. The uncertainty in measurements of bubble resonance using this technique is between 0.5 and $2 \%{ }^{19}$. The $\omega_{\mathrm{i}} \pm\left(\omega_{\mathrm{p}} / 2\right)$ scattering would be expected to be sharper, since it is a non-linear effect of higher order than the scattering of the $\omega_{i} \pm \omega_{\mathrm{p}}$ frequency, and so would fall off as one moves away from resonance faster than the sum and difference scattering do. An alternative view is that the damping of the subharmonic is less: its decay time is longer, so that in the frequency domain its spread will be less. Although no analysis of the damping of non-linear combination frequencies is available, Eller $^{20}$ calculated the damping of forced radial oscillations for a 

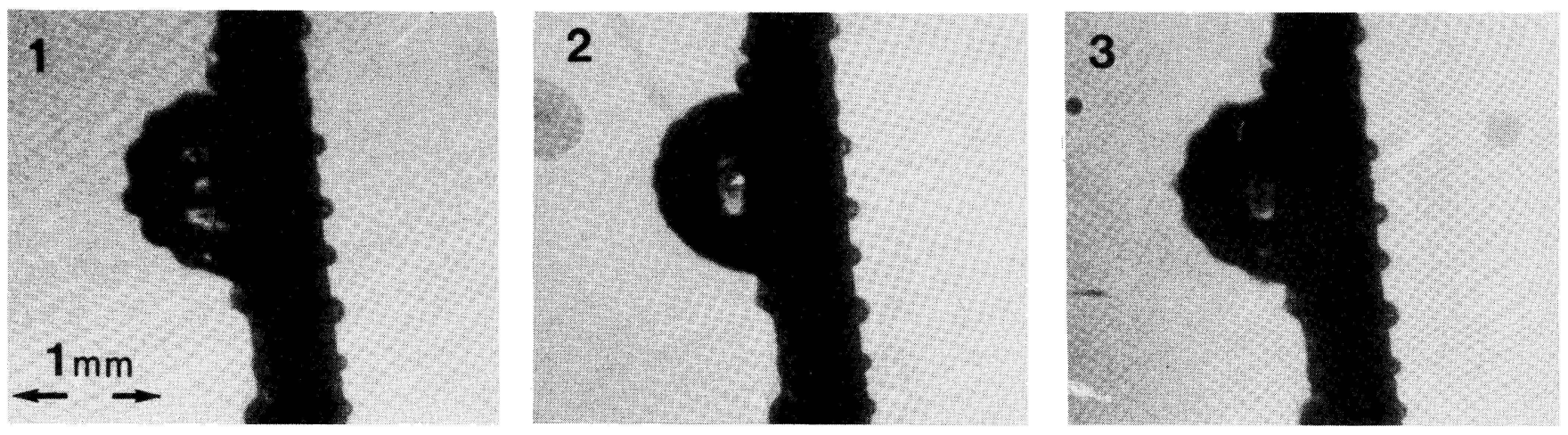

Figure 7 Selected frames from a high speed sequence showing a bubble of diameter $\approx 1 \mathrm{~mm}$ attached to a wire and subjected to acoustic waves at a pump frequency of $4.370 \mathrm{kHz}$. The oscillating ripples on the bubble surface are pronounced in frames 1 and 3 but greatly reduced in frame 2. Small bubbles have formed on the wire surface. Frame rate 6000 f.p.s.

bubble insonated by a single frequency. The damping of any subharmonic signal is not discussed. However, it is interesting to compare the damping of the bubble of radius $0.645 \mathrm{~mm}$ shown in Figure 4, when insonated at $4.90 \mathrm{kHz}$ (resonance) and at $2.45 \mathrm{kHz}$ (the frequency of the subharmonic of the resonance). Following Eller's calculation, small amplitude pulsations of the bubble can be expressed as

$m \ddot{v}+b \dot{v}+k v=-P_{\mathrm{A}} \mathrm{e} i^{\omega t}$

where $v$ is the change in volume and $m$ the effective mass of the bubble. The right-hand side of Equation (5) represents the acoustic driving term. The damping term, $b$, is the sum of thermal, viscous and radiation components. Eller ${ }^{20}$ provided expressions for calculating the damping constant, $d$, where

$d=\omega b / k$

For an air bubble of radius $0.645 \mathrm{~mm}$ in water, the damping constant at $4.90 \mathrm{kHz}$ has a value of $4.40 \times 10^{-2}$. At an insonation frequency of $2.45 \mathrm{kHz}$, the value equals $4.35 \times 10^{-2}$. A decay time can be represented by $1 /(\omega d)$, and so decay times for the bubble are 0.74 and $1.49 \mathrm{~ms}$ at insonation frequencies of 4.90 and $2.45 \mathrm{kHz}$, respectively. The factor of $\approx 2$ difference in decay times arises almost entirely from the change in $\omega$, since the value of $d$ varies here by only $\approx 1 \%$. Similar calculations for the range of bubble sizes studied suggest that $d$ does not vary by more than a few per cent for insonation at the resonance, and at half the resonance, frequency. The decay time at half-resonance will therefore tend to be twice that for insonation at resonance [equation (6)] and this might well explain why the spread of the $\omega_{\mathrm{i}} \pm\left(\omega_{\mathrm{p}} / 2\right)$ signal is $40 \pm 20 \%$ that of the $\omega_{\mathrm{i}} \pm \omega_{\mathrm{p}}$ signal.

The non-linear scattering from the bubble produced signals not just at $\omega_{\mathrm{i}} \pm \omega_{\mathrm{p}}$ and $\omega_{\mathrm{i}} \pm\left(\omega_{\mathrm{p}} / 2\right)$, but also at combination frequencies of the imaging signal and superharmonics of the pump. Though these tended to be even sharper than the $\omega_{i} \pm\left(\omega_{\mathrm{p}} / 2\right)$ response, their low amplitude made them unsuitable for sizing purposes. They are not visible in Figure 2 as they lie outside the range of the frequencies illustrated.

This technique can be used to indicate bubble coalescence or fragmentation through a comparison of the response curves for both increasing and decreasing values of pump frequency ${ }^{19}$. The value of $\kappa$ for this process can be estimated from Figure 5. Best-fit lines to the data give values for $\kappa$ of $1.05 \pm 0.04$ for air, $1.15 \pm 0.11$ for helium and $0.995 \pm 0.034$ for propane. All, therefore, tend towards the isothermal.

The four-bubble array illustrated in Figure 3 was successfully resolved (Figure 6). Using $\kappa=1.05$ the peaks at 4.65 and $4.38 \mathrm{kHz}$ correspond to bubble radii of 0.61 and $0.64 \mathrm{~mm}$, respectively, indicating that this system is capable of resolving bubbles differing in radius by only $30 \mu \mathrm{m}$. This represents the resolution of two bubbles whose radii differ by $5 \%$.

\section{Conclusions}

When a bubble is subjected to an audio pump signal, $\omega_{\mathrm{p}}$, and a megahertz imaging signal, $\omega_{i}$, the scattered acoustic signals contain additional frequencies that become increasingly strong as the pump approaches the bubble resonance. In particular, signals are present at $\omega_{\mathrm{i}} \pm \omega_{\mathrm{p}}$ and $\omega_{\mathrm{i}} \pm\left(\omega_{\mathrm{p}} / 2\right)$, the latter tending to a sharper response and so being more suitable for bubble sizing and resolution. The technique has been used to resolve bubbles (of radii $\approx 0.6 \mathrm{~mm}$ ) a distance of $5 \mathrm{~mm}$ apart, differing in radius by $\approx 5 \%$. It is, therefore, a promising method for bubble sizing.

\section{Acknowledgements}

T.G. Leighton wishes to thank Magdalene College, Cambridge and the Science and Engineering Research Council for Research Fellowships. The authors wish to thank K. Fagan for his help with the photography, Dr W. Fitzgerald and Dr N. A. Safford for helpful suggestions, and Marconi Maritime for a grant to the laboratory.

\section{References}

1 Neppiras, E.A. Acoustic cavitation Phys Rep (1980) 61 159-251

2 Minnaert, $\mathbf{M}$. On musical air-bubbles and sounds of running water Phil Mag (1933) 16 235-248

3 Leighton, T.G. and Walton, A.J. An experimental study of the sound emitted from gas bubbles in a liquid Eur J Phys (1987) 8 98-104

4 Pumphrey, H.C. and Walton, A.J. Experimental study of the sound emitted by water drops impacting on a water surface Eur $J$ Phys (1988) $9225-231$

5 Fairbank, W.M. and Scully, M.O. A new noninvasive technique for cardiac pressure measurement: resonant scattering of ultrasound from bubbles IEEE Trans Biomed Eng (1977) 24 107-110 
6 Nishi, R.Y. Ultrasonic detection of bubbles with Doppler flow transducers Ultrasonics (1972) 10 173-179

7 Miller, D.L. Ultrasonic detection of resonant cavitation bubbles in a flow tube by their second-harmonic emissions Ultrasonics (1981) 19 217-224

8 Shankar, P.M., Chapelon, J.Y. and Newhouse, V.L. Fluid pressure measurement using bubbles insonified by two frequencies Ultrasonics (1986) 24 333-336

9 Belcher, E.O. Quantification of bubbles formed in animals and man during decompression IEEE Trans Biomed Eng (1980) 27 $330-338$

10 Kisman, H. Spectral analysis of Doppler ultrasonic decompression data Ultrasonics (1977) 15 105-110

11 Tickner, E.G. Precision microbubbles for right side intercardiac pressure and flow measurements, in: Contrast Echocardiography (Eds Meltzer, R.S. and Roeland, J.) Nijhoff, London, UK (1982)

12 Watkins, R.D., Barrett, L.M. and McKnight, J.A. Ultrasonic waveguide for use in the sodium coolant of fast reactors $\mathrm{Nucl}$ Energy (1988) 27 85-89

13 Hulshof, H.J.M. and Schurink, F. Continuous ultrasonic waves to detect steam bubbles in water under high pressure Kema Scientific and Technical Reports (1985) 3 61-69

14 Gross, D.R., Miller, D.L. and Williams, A.R. A search for ultrasonic cavitation within the canine cardiovascular system Ultrasound Med Biol (1985) 11 85-97

15 Newhouse, V.L. and Shankar, P.M. Bubble size measurement using the nonlinear mixing of two frequencies $J$ Acoust Soc Am (1984) 75 1473-1477

16 Main, I.G. Vibrations and Waves in Physics 2nd Edn, Cambridge University Press, Cambridge, UK (1984) 104-107

17 Walton, A.J. and Reynolds, G.T. Sonoluminescence Adv Phys (1984) 33 595-660

18 Bohn, L. Acoustic pressure variation and the spectrum in oscillatory cavitation Acustica (1957) 7 201-216

19 Leighton, T.G., Lingard, R.J., Walton, A.J. and Field, J.E. Bubble sizing by the nonlinear scattering of two acoustic frequencies, in: Natural Physical Sources of Underwater Sound (Ed Kerman, B.) Kluwer Academic Publishers, Dordrecht, The Netherlands (1990)

20 Eller, A.I. Damping constants of pulsating bubbles $J$ Acoust Soc Am (1970) 47 1469-1470 